\title{
A new extension of quantum Simpson's and quantum Newton's type inequalities for quantum differentiable convex functions
}

\author{
Muhammad Aamir $\mathrm{Ali}^{1}$, Hüseyin BUDAK${ }^{2}$, and Zhiyue Zhang ${ }^{1}$ \\ ${ }^{1}$ Nanjing Normal University School of Mathematical Sciences \\ ${ }^{2}$ Düzce University
}

February 22, 2021

\begin{abstract}
In this paper, we prove two identities involving quantum derivatives, quantum integrals, and certain parameters. Using the newly proved identities, we prove new inequalities of Simpson's and Newton's type for quantum differentiable convex functions under certain assumptions. Moreover, we discuss the special cases of our main results and obtain some new and existing Simpson's type inequalities, Newton's type inequalities, midpoint type inequalities and trapezoidal type inequalities.
\end{abstract}

\section{Hosted file}

MMAS11521.pdf available at https://authorea.com/users/330633/articles/510254-a-newextension-of-quantum-simpson-s-and-quantum-newton-s-type-inequalities-for-quantumdifferentiable-convex-functions 